Research Paper

\title{
Laparoscopic gastrectomy reduces adverse postoperative outcomes and decreases morbidity for gastric cancer patients with visceral obesity: a propensity score-matched analysis
}

Chenchen Mao, MD ${ }^{1 \# ; ~ X i a o d o n g ~ C h e n, ~ M D ~}{ }^{1 \# ; ~ X i a n g w e i ~ S u n, ~ M D ~}{ }^{1 \# ; ~ X i a n g ~ W a n g, ~ M D ~}{ }^{1}$; Ce Zhu, MD ${ }^{1}$; Wenjing Chen, $\mathrm{MD}^{2}$; Xiangyang Xue, MD, $\mathrm{PhD}^{3 凶}$; Xian Shen, MD, $\mathrm{PhD}^{1,2 \bowtie}$

1. Department of Gastrointestinal Surgery, The Second Affiliated Hospital, Wenzhou Medical University, Wenzhou, Zhejiang, China.

2. Department of Gastrointestinal Surgery, The First Affiliated Hospital, Wenzhou Medical University, Wenzhou, Zhejiang, China.

3. Department of Medical Microbiology and Immunology, Basic Medical College, Wenzhou Medical University, Wenzhou, Zhejiang, China.

\#These authors contributed equally to this work.

$\square$ Corresponding authors: Xian Shen, MD, PhD, Department of Gastrointestinal Surgery, The Second Affiliated Hospital, Wenzhou Medical University, Wenzhou, Zhejiang Province, China. E-mail: shenxian5166@gmail.com; Xiangyang Xue, MD, PhD, Department of Microbiology and Immunology, School of Basic Medical Sciences, Institute of Molecular Virology and Immunology, Institute of Tropical Medicine, Wenzhou Medical University, Wenzhou, China. E-mail: wzxxy001@163.com.

(c) The author(s). This is an open access article distributed under the terms of the Creative Commons Attribution License (https://creativecommons.org/licenses/by/4.0/). See http://ivyspring.com/terms for full terms and conditions.

Received: 2020.04.28; Accepted: 2021.02.17; Published: 2021.02.21

\begin{abstract}
Background: Laparoscopic gastrectomy for gastric cancer shortens the recovery period without decreasing long-term survival. However, clinical evidence on whether laparoscopic radical gastrectomy reduces the surgical stress and improves the short- and long-term outcomes of obese patients with gastric cancer is lacking. We compared the short- and long-term outcomes of gastric cancer patients with visceral obesity (VO) who underwent laparoscopic gastrectomy (LG) or open gastrectomy (OG).

Methods: We prospectively collected data from 578 patients who underwent radical gastrectomy in two centers between January 2014 and December 2016. The visceral fat area (VFA) was measured on the umbilicus level, and VFA $\geq 100 \mathrm{~cm}^{2}$ was defined as VO. The section bias was reduced by conducting a propensity score matching analysis. The short- and long-term outcomes were further compared between patients who underwent $O G$ and those who underwent $L G$.

Results: Overall, 245 patients (42.61\%) were classified as having VO, of whom 102 were included for further analysis after matching. There were no significant differences in clinical characteristics between the two groups in the matched cohort. The LG group had significantly fewer overall complications $(\mathrm{P}<0.001)$ and shorter postoperative hospital stays $(\mathrm{P}<0.001)$. Subgroup analysis of postoperative complications also showed that the incidence of surgical complications was lower in the LG group $(P=0.002)$. Further survival analysis showed the $L G$ group had significantly better long-term overall survival $(P=0.017)$.

Conclusions: Compared with open radical gastrectomy, laparoscopy would reduce the rate of postoperative complications in patients with VO, as well as prolong their overall survival.
\end{abstract}

Key words: obesity, abdominal; laparoscopy; stomach neoplasms; postoperative complications; survival

\section{Introduction}

Gastric cancer (GC) is the fourth most common cancer worldwide and the third leading cause of cancer-related mortality, with 723,000 deaths annually $[1,2]$. Obesity has been reported to be associated with poor surgical outcomes in GC, including fewer lymph node dissections and more postoperative complications $[3,4]$. Traditionally, body mass index (BMI) has been broadly used to indicate obesity $[5,6]$. 
However, BMI cannot distinguish fat distribution within the intra-abdominal cavity or different types of adipose tissue [7]. Several studies recently proposed that visceral fat is likely to be a more optimal tool for predicting surgical outcomes, and visceral obesity (VO) is superior to BMI for the prediction of complications after colonic and gastric surgery [8-11]. Therefore, studies should examine the amount of body fat and use VO in clinical practice and research.

Laparoscopy-assisted gastrectomy (LG) has been rapidly adopted for GC [12]. Multiple studies have shown that compared to traditional open surgery, laparoscopic radical gastrectomy has the apparent advantage of minimizing invasive procedures and shortening the recovery period; laparoscopy also results in comparable long-term survival [13-15]. However, the thicker abdominal wall in obese individuals increases the operation difficulty and postoperative infection risk. Studies have also shown that obesity may be a contraindication for laparoscopic surgery [4]. There is still a lack of clinical evidence whether laparoscopic radical gastrectomy can reduce the surgical stress and improve the shortand long-term outcomes of obese patients with GC.

The purpose of our study was to provide a reference for the clinical implementation of the best surgical approach by comparing and analyzing the short- and long-term outcomes of GC patients with $\mathrm{VO}$ who underwent different surgical procedures.

\section{Materials and methods}

\section{Study design and patient population}

Data were prospectively collected from patients who underwent R0 gastrectomy and D2 lymphadenectomy at the Gastrointestinal Surgical Departments of the Second Affiliated Hospital of Wenzhou Medical University and the First Affiliated Hospital of Wenzhou Medical University in China between January 2014 and December 2016. The choice of open or laparoscopic surgery was based on the doctor's advice and patient's decision after reading the informed consent form. This study was approved by the ethics committees of the Second Affiliated Hospital of Wenzhou Medical University, and all participants provided written informed consent prior to study participation.

\section{Inclusion/exclusion criteria}

All adult patients who met the following criteria were included in the analysis: (a) patients with histopathologically confirmed gastric adenocarcinoma and scheduled to undergo radical gastrectomy; (b) patients aged $\geq 18$ years; and (c) patients who provided written informed consent to participate in the study. The following exclusion criteria were applied: (a) patients who lacked imaging data; (b) patients who underwent palliative surgery or emergency surgery; (c) patients who received neoadjuvant chemotherapy or radiotherapy; (d) patients who had a severe immune, blood, or endocrine disease; (e) patients with GC concurrent with other malignant tumors; and (f) patients with substantial absence of clinical data. Operations were performed per the Japanese Gastric Cancer Treatment Guidelines 2010, version 3.

\section{Baseline data collection}

All data were collected prospectively and maintained in a digital database. For each patient enrolled in this study, demographic details, including age, sex, BMI, American Society of Anesthesiologists (ASA) grade, abdominal operation history, and NRS 2002 score were collated. Other details pertaining to the operation, such as tumor location, tumor differentiation, pathological classification, and histopathologic staging according to the TNM staging (AJCC Cancer Staging System, 8th $^{\text {th }}$ ed), were also collected. Additionally, postoperative outcomes according to the Clavien-Dindo classification [16], postoperative hospital stays, hospitalization costs, and overall survival (OS) data were also collected.

\section{Computed tomography-based measurement of visceral fat area}

Preoperatively, all patients underwent computed tomography of the general abdominal cavity. We selected a single cross-section scan at the umbilicus level for quantification of the degree of visceral fat. A threshold of -140 to -50 was used for visceral fat, comparable to the methods in previous studies [17-19]. The total fat area was calculated using a dedicated processing system (version 3.0.11.3, BN17 32-bit; INFINITT Healthcare Co., Ltd., Seoul, South Korea). Patients with a visceral fat area (VFA) larger than $100 \mathrm{~cm}^{2}$ were classified as having $\mathrm{VO}$, as suggested in the previous literature $[6,11,20]$.

\section{Follow-up}

Trained doctors were responsible for visiting the patients and conducting a phone call to follow up with the patients after surgery. The last follow-up evaluation was conducted in January 2019. Overall survival (OS) was defined from the day of surgery until death or until the final follow-up date in January 2019, whichever was first.

\section{Statistical analyses}

To compare the laparoscopic group and the open group, propensity scores were generated using a logistic regression model on the all the baseline covariates: age, BMI, NRS score, ASA grade, 
hypertension history, diabetes mellitus history, abdominal surgery history, tumor location, TNM stage, differentiated degree, pathological type, and combined resection. Propensity score matching (PSM) was performed in a 1:1 ratio, and an optimal matching with a caliper size of 0.03 was used to avoid poor matches. The two matched groups were evaluated with respect to the study endpoints. Means and standard deviations were used for all continuous data, and numbers and percentages were calculated for all categorical data. In univariate analyses, the independent t-test and Mann-Whitney U-test were used to analyze intergroup differences in continuous variables. The chi-square test and Fisher's exact test were applied to categorical variables. In multivariate analyses, conditional logistic regression analyses were performed to evaluate the association between patient characteristics and short-term outcomes. OS was defined as the time between the date of diagnosis and the date of death or last known follow-up. The Kaplan-Meier method and log-rank test were used to estimate and compare survival, respectively, based on specific factors. The Cox proportional hazard model was used to estimate the risk ratio in univariate and multivariate analyses, and results were expressed as odds ratios (ORs) with $95 \%$ confidence intervals (CIs). All $\mathrm{P}$ values were two-sided, and $\mathrm{P}<0.05$ was considered statistically significant. All statistical analyses were performed using IBM SPSS Statistics for Windows/Macintosh, version 22.0 (IBM Corp., Armonk, N.Y., USA) and R version 3.0.1 (https://www.r-project.org).

\section{Results}

\section{Patient characteristics}

Among the initially recruited 578 patients, 15 were subsequently excluded, and the reasons for exclusion are detailed in Figure 1. Of the 563 remaining patients, 245 had VO and were therefore included in the study.

As summarized in Table 1, laparoscopic surgery and open surgery were performed in 57 patients $(23.27 \%)$ and 188 patients $(76.73 \%)$, respectively. LG was more likely performed in patients with lower TNM stage $(\mathrm{P}=0.006)$. Further, patients who underwent laparoscopic surgery were significantly younger $(\mathrm{P}=0.003)$ and had lower NRS scores $(\mathrm{P}=0.039)$ and ASA stage $(\mathrm{P}=0.013)$ than patients who underwent open surgery. There was no significant difference in sex, BMI, the incidence of hypertension or diabetes mellitus, abdominal surgery history, tumor location, tumor differentiation, pathological classification, or combined organ resection between the two groups. After PSM, the total cohort comprised
102 patients: 51 patients comprised the laparoscopic gastrectomy (LG) group and 51 patients comprised the open gastrectomy (OG) group. As shown in Table 1 , the two groups of patients were well matched, and there were no significant differences between the groups after PSM.

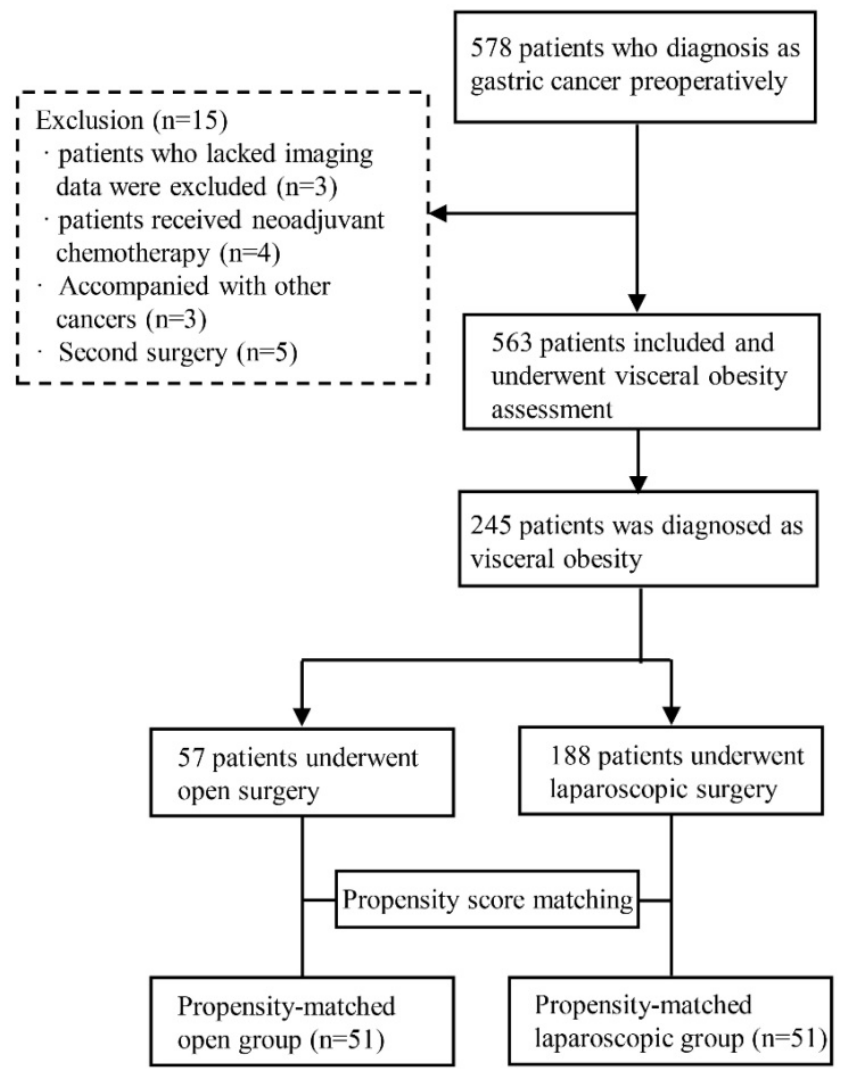

Figure 1. Flow chart of the study procedure.

\section{Laparoscopic surgery was an independent protective factor against postoperative complications}

In the matched cohort, 28 of 102 patients $(27.45 \%)$ experienced postoperative complications (Table 2). The postoperative complication rate was significantly lower in the LG group than in the OG group $(11.76 \%$ versus $43.14 \%, \quad \mathrm{P}<0.001)$. Further analysis of complications between the two groups showed that the rate of surgical complications was significantly lower in the LG group than in the OG group $(5.88 \%$ versus $29.41 \%, P=0.002)$. Other complications were also lower in the LG group, but no statistical significance was found. Additionally, no patients suffered 30-day mortality, and only one had a second operation after open gastrectomy. There were no significant differences between the two groups of patients in the type of surgery. However, more patients underwent Billroth-I reconstruction in the laparoscopic group than in the open group (Table 2). 
Table 1. Patient baseline characteristics

\begin{tabular}{|c|c|c|c|c|c|c|}
\hline \multirow[t]{2}{*}{ Factors } & \multicolumn{3}{|c|}{ Unmatched comparison } & \multicolumn{3}{|c|}{ Unmatched comparison } \\
\hline & Laparoscopic $(\mathrm{n}=57)$ & Open $(n=188)$ & $P$ & Laparoscopic $(\mathrm{n}=51)$ & Open $(n=51)$ & $P$ \\
\hline Gender & & & 0.652 & & & 0.799 \\
\hline Male & $45(78.95 \%)$ & $143(76.06 \%)$ & & $42(82.35 \%)$ & $41(80.39 \%)$ & \\
\hline Female & $12(21.05 \%)$ & $45(23.94 \%)$ & & $9(17.65 \%)$ & $10(19.61 \%)$ & \\
\hline Age (y) & & & $0.003^{*}$ & & & 0.074 \\
\hline$\leq 65$ & $35(61.40 \%)$ & $74(39.36 \%)$ & & $32(62.75 \%)$ & $23(45.10 \%)$ & \\
\hline$>65$ & $22(38.60 \%)$ & $114(60.64 \%)$ & & $19(37.25 \%)$ & $28(54.90 \%)$ & \\
\hline BMI $\left(\mathrm{kg} / \mathrm{m}^{2}\right)$ & & & 0.434 & & & 0.427 \\
\hline$\leq 18.5$ & $0(0 \%)$ & $3(1.60 \%)$ & & $0(0 \%)$ & $0(0 \%)$ & \\
\hline $18.5-24$ & $27(47.37 \%)$ & $84(44.68 \%)$ & & $26(50.98 \%)$ & $22(43.14 \%)$ & \\
\hline$>24$ & $30(52.63 \%)$ & $101(53.72 \%)$ & & $25(49.02 \%)$ & $29(56.86 \%)$ & \\
\hline NRS 2002 score & & & $0.039^{*}$ & & & 0.822 \\
\hline $1-2$ & $45(78.95 \%)$ & $120(63.83 \%)$ & & $40(78.43 \%)$ & $40(78.43 \%)$ & \\
\hline $3-4$ & $11(19.30 \%)$ & $52(27.66 \%)$ & & $10(19.61 \%)$ & $9(17.65 \%)$ & \\
\hline $5-6$ & $1(1.75 \%)$ & $16(8.51 \%)$ & & $1(1.96 \%)$ & $2(3.92 \%)$ & \\
\hline ASA grade & & & $0.013^{*}$ & & & 1.000 \\
\hline $1-2$ & $52(91.23 \%)$ & $143(76.06 \%)$ & & $46(90.20 \%)$ & $47(92.16 \%)$ & \\
\hline $3-4$ & $5(8.77 \%)$ & $45(23.94 \%)$ & & $5(9.80 \%)$ & $4(7.84 \%)$ & \\
\hline Hypertension & & & 0.856 & & & 1.000 \\
\hline Yes & $21(36.84 \%)$ & $68(36.17 \%)$ & & $21(41.18 \%)$ & $21(41.18 \%)$ & \\
\hline No & $36(63.1619 .30 \%)$ & $120(63.83 \%)$ & & $30(58.82 \%)$ & $30(58.82 \%)$ & \\
\hline Diabetes mellitus & & & 0.880 & & & 1.000 \\
\hline Yes & $11(19.30 \%)$ & $38(20.21 \%)$ & & $9(17.65 \%)$ & $9(17.65 \%)$ & \\
\hline No & $46(80.70 \%)$ & $150(79.79 \%)$ & & $42(82.35 \%)$ & $42(82.35 \%)$ & \\
\hline Previous abdominal surgery & & & 0.361 & & & 0.433 \\
\hline Yes & $5(8.77 \%)$ & $25(13.30 \%)$ & & $5(9.80 \%)$ & $2(3.92 \%)$ & \\
\hline No & $52(91.23 \%)$ & $163(86.70 \%)$ & & $46(90.20 \%)$ & $49(96.08)$ & \\
\hline Tumor location & & & 0.480 & & & \\
\hline Cardia & $8(14.04 \%)$ & $30(15.96 \%)$ & & $5(9.80 \%)$ & $6(11.76 \%)$ & 0.688 \\
\hline Body & $15(26.32 \%)$ & $33(17.55 \%)$ & & $13(25.49 \%)$ & $13(25.49 \%)$ & \\
\hline Antrum & $33(57.89 \%)$ & $118(62.77 \%)$ & & $32(62.75 \%)$ & $32(62.75 \%)$ & \\
\hline Total & $1(1.75 \%)$ & $7(3.72 \%)$ & & $1(1.96 \%)$ & $0(0 \%)$ & \\
\hline Differentiated degree & & & 0.952 & & & 0.573 \\
\hline Differentiated & $44(77.19 \%)$ & $142(75.53 \%)$ & & $39(76.47 \%)$ & $35(68.63 \%)$ & \\
\hline Undifferentiated & $5(8.77 \%)$ & $19(10.11 \%)$ & & $5(9.80 \%)$ & $5(9.80 \%)$ & \\
\hline Signet ring carcinoma & $8(14.04 \%)$ & $27(14.36 \%)$ & & $7(13.73 \%)$ & $11(21.57 \%)$ & \\
\hline Pathological type & & & 0.848 & & & 1.000 \\
\hline Ulcerative type & $53(92.98 \%)$ & $172(91.49 \%)$ & & $47(92.16 \%)$ & $46(90.20 \%)$ & \\
\hline Non-ulcerative type & $4(7.02 \%)$ & $16(8.51 \%)$ & & $4(7.84 \%)$ & $5(9.80 \%)$ & \\
\hline T stage & & & 0.028 & & & 0.349 \\
\hline I & $22(38.60 \%)$ & $39(20.74 \%)$ & & $23(45.11 \%)$ & $16(31.37 \%)$ & \\
\hline II & $9(15.79 \%)$ & $29(15.53 \%)$ & & $6(11.76 \%)$ & $12(23.53 \%)$ & \\
\hline III & $6(10.53 \%)$ & $38(20.21 \%)$ & & $6(11.76 \%)$ & $6(11.76 \%)$ & \\
\hline IV & $19(33.33 \%)$ & $82(43.62 \%)$ & & $16(31.37 \%)$ & $17(33.34 \%)$ & \\
\hline Lymphatic metastasis number & & & 0.043 & & & 0.204 \\
\hline 0 & $35(61.40 \%)$ & $82(43.62 \%)$ & & $31(60.78 \%)$ & $27(52.94 \%)$ & \\
\hline $1-2$ & $10(17.54 \%)$ & $35(18.61 \%)$ & & $9(17.65 \%)$ & $10(19.61 \%)$ & \\
\hline $3-6$ & $9(15.79 \%)$ & $38(20.21 \%)$ & & $9(17.65 \%)$ & $8(15.69 \%)$ & \\
\hline $7-16$ & $3(5.3 \%)$ & $25(13.30 \%)$ & & $2(3.92 \%)$ & $2(3.92 \%)$ & \\
\hline$>16$ & $0(0 \%)$ & $7(3.72 \%)$ & & $0(0 \%)$ & $4(7.84 \%)$ & \\
\hline TNM stage & & & 0.006 & & & 0.924 \\
\hline I & $29(50.88 \%)$ & $53(28.19 \%)$ & & $25(49.02 \%)$ & $23(45.10 \%)$ & \\
\hline II & $11(19.30 \%)$ & $52(27.66 \%)$ & & $11(21.57 \%)$ & $12(23.53 \%)$ & \\
\hline III & $17(29.82 \%)$ & $83(44.15 \%)$ & & $15(29.41 \%)$ & $16(31.37 \%)$ & \\
\hline Combined organ resection & & & 0.387 & & & 1.000 \\
\hline Yes & $2(3.51 \%)$ & $15(7.98 \%)$ & & $2(3.92 \%)$ & $1(1.96 \%)$ & \\
\hline No & $55(96.49 \%)$ & $173(92.02 \%)$ & & $49(96.08 \%)$ & $50(98.04 \%)$ & \\
\hline Postoperative chemotherapy & & & 0.007 & & & 0.842 \\
\hline Yes & $30(52.63 \%)$ & $135(71.81 \%)$ & & $29(56.86 \%)$ & $28(54.90 \%)$ & \\
\hline No & $27(47.37 \%)$ & $53(28.19 \%)$ & & $22(43.14 \%)$ & $23(45.10 \%)$ & \\
\hline
\end{tabular}

BMI; body mass index, ASA; American Society of Anesthesiologists, NRS 2002; nutritional risk screening 2002, TNM tumor-node-metastasis;

The values represent the number of patients, and values in parentheses represent percentages;

*Represents $P<0.05$, which was considered to be statistically significant. 
Table 2. Surgical outcomes before and after matching

\begin{tabular}{|c|c|c|c|c|c|c|}
\hline \multirow[t]{2}{*}{ Factors } & \multicolumn{3}{|c|}{ Unmatched comparison } & \multicolumn{3}{|l|}{ Matched comparison } \\
\hline & LG (n=57) & OG $(n=188)$ & $P$ & LG $(n=51)$ & $\mathrm{OG}(\mathrm{n}=51)$ & $P$ \\
\hline Total complicationsa & $7(12.28 \%)$ & $74(39.36 \%)$ & $<0.001^{*}$ & $6(11.76 \%)$ & $22(43.14 \%)$ & $<0.001^{*}$ \\
\hline \multicolumn{7}{|l|}{ Clavien-Dindo grade } \\
\hline Grade I & $2(3.51 \%)$ & $8(4.26 \%)$ & 1.000 & $1(1.96 \%)$ & $4(7.84 \%)$ & 0.359 \\
\hline Grade II & $4(7.02 \%)$ & $44(23.40 \%)$ & $0.006^{*}$ & $4(7.84 \%)$ & $14(27.45 \%)$ & $0.009^{*}$ \\
\hline Grade III & $1(1.75 \%)$ & $14(7.45 \%)$ & 0.209 & $1(1.96 \%)$ & $2(3.92 \%)$ & 1.000 \\
\hline Grade IV & $0(0.00 \%)$ & $8(4.26 \%)$ & 0.247 & $0(0.00 \%)$ & $2(3.92 \%)$ & 0.475 \\
\hline Severe complications ${ }^{b}$ & $1(1.75 \%)$ & $22(11.71 \%)$ & 0.024 & $1(1.96 \%)$ & $4(7.84 \%)$ & 0.359 \\
\hline \multicolumn{7}{|l|}{ Detail of complications } \\
\hline Surgical complications & $3(5.26 \%)$ & $47(25.00 \%)$ & $0.001^{*}$ & $3(5.88 \%)$ & $15(29.41 \%)$ & $0.002^{*}$ \\
\hline Gastrointestinal dysfunction & $0(0.00 \%)$ & $7(3.72 \%)$ & 0.306 & $0(0.00 \%)$ & $5(9.80 \%)$ & 0.067 \\
\hline Intestinal obstruction & $0(0.00 \%)$ & $5(2.66 \%)$ & 0.478 & $0(0.00 \%)$ & $2(3.92 \%)$ & 0.475 \\
\hline Anastomotic leakage & $0(0.00 \%)$ & $5(2.66 \%)$ & 0.478 & $0(0.00 \%)$ & $2(3.92 \%)$ & 0.475 \\
\hline Severe wound infection & $0(0.00 \%)$ & $4(2.13 \%)$ & 0.576 & $0(0.00 \%)$ & $1(1.96 \%)$ & 1.000 \\
\hline Intra-abdominal infection & $2(3.51 \%)$ & $19(10.11 \%)$ & 0.198 & $2(3.92 \%)$ & $3(5.88 \%)$ & 1.000 \\
\hline Intra-abdominal Bleeding & $1(1.75 \%)$ & $7(3.72 \%)$ & 0.759 & $1(1.96 \%)$ & $2(3.92 \%)$ & 1.000 \\
\hline Medical complications & $4(7.02 \%)$ & $27(14.36 \%)$ & 0.144 & $3(5.88 \%)$ & $7(13.73 \%)$ & 0.183 \\
\hline Pleural and peritoneal effusion & $4(7.02 \%)$ & $11(5.85 \%)$ & 0.995 & $3(5.88 \%)$ & $4(7.84 \%)$ & 1.000 \\
\hline Pulmonary complications & $0(0.00 \%)$ & $8(4.26 \%)$ & 0.247 & $0(0.00 \%)$ & $1(1.96 \%)$ & 1.000 \\
\hline Venous thrombosis & $0(0.00 \%)$ & $8(4.26 \%)$ & 0.247 & $0(0.00 \%)$ & $2(3.92 \%)$ & 0.475 \\
\hline 30-day mortality & $0(0.00 \%)$ & $3(1.60 \%)$ & 1.000 & $0(0.00 \%)$ & $0(0.00 \%)$ & - \\
\hline Operative time, $(X \pm S D)$, min & $227.87 \pm 47.47$ & $200.46 \pm 49.42$ & $<0.001^{*}$ & $226.29 \pm 48.74$ & $191.25 \pm 52.23$ & $0.001^{*}$ \\
\hline Postoperative hospital stays, $(X \pm S D)$, days & $11.67 \pm 3.87$ & $17.12 \pm 9.25$ & $<0.001^{*}$ & $11.73 \pm 4.03$ & $17.45 \pm 9.28$ & $<0.001^{*}$ \\
\hline Hospitalization costs, $(X \pm S D)$, yuan & $62574.56 \pm 18724.56$ & $66928.08 \pm 35050.36$ & 0.370 & $62763.13 \pm 19705.45$ & $63020.28 \pm 28839.08$ & 0.958 \\
\hline
\end{tabular}

Values are shown as $\mathrm{n}(\%)$ unless otherwise indicated.

aPostoperative complications in this study were defined as any adverse event corresponding to Clavien-Dindo classification grade, occurring within 30 days after surgery. If a patient had more than one type of complication, the complication with the highest grade was used for the analysis.

bClavien-Dindo grade $\geq$ III.

${ }^{*} P<0.05$, statistically significant.

Univariate and multivariate analyses of factors associated with overall postoperative complications are summarized in Table 3. In the univariate analysis, overall postoperative complications were significantly associated with laparoscopic surgery $(\mathrm{P}=0.001)$. Further multivariate logistic regression analysis also showed that laparoscopic surgery was an independent protective factor for postoperative complications (OR 0.141, 95\% CI 0.042-0.472, P=0.001).

\section{Laparoscopic surgery was independently associated with better OS}

The mean follow-up was 33.92 ( \pm 11.32$)$ months. There was no significant difference in the follow-up period between the LG and OG groups (35.36 \pm 8.35 versus $32.47 \pm 13.60$ months, $P=0.200$ ). As shown in Figure 1, patients in the LG group had a better outcome than that in the OG group $(\mathrm{P}=0.017)$.

Further evaluation of the potential factors influencing OS was performed. A univariate analysis showed that OS was affected by a higher NRS score (NRS 3-4: HR 1.698, 95\% CI 0.540-5.341, $\mathrm{P}=0.366$; NRS 5-6: HR 6.180, 95\% CI 1.361-28.055, $\mathrm{P}=0.018)$, TNM stage (TNM II: 4.758, 95\% CI 0.871-25.995, $\mathrm{P}=0.072$, TNM III: $10.492,95 \%$ CI 2.321-47.434, $\mathrm{P}=0.002$ ), and the type of surgery (Laparoscopy: HR $0.280,95 \%$ CI 0.091-0.859, $\mathrm{P}=0.026$ ). A multivariate analysis showed that a higher NRS score (NRS 3-4: HR 2.588, 95\% CI 0.579-11.570, $\mathrm{P}=0.213$; NRS 5-6: HR 12.968, 95\% CI
1.780-94.495, $\mathrm{P}=0.011)$ and TNM stage (TNM II: 5.686, 95\% CI 0.737-43.861, P=0.095, TNM III: $17.492,95 \%$ CI 2.445-125.157, $\quad \mathrm{P}=0.004) \quad$ were independently associated with worse OS, whereas laparoscopic surgery (HR $0.264,95 \%$ CI $0.077-0.905, \mathrm{P}=0.034$ ) was an independent protective factor (Table 4 ).

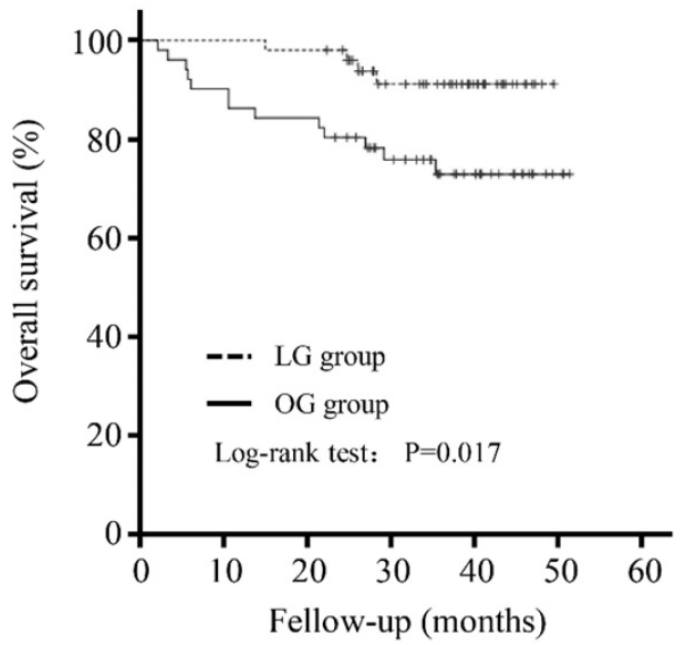

Figure 2. Five-year overall survival curve calculated using the Kaplan-Meier method comparing the LG and OG groups.

\section{Discussion}

In the current study, we first tried to specifically compare the effect of LG and OG on the surgical 
outcomes in GC patients with VO. Interestingly, compared with open radical gastrectomy, laparoscopy gastrectomy would reduce the rate of postoperative complications in patients with $\mathrm{VO}$ and prolong their OS.

According to a recent study, the number of overweight or obese people has already surpassed the number of underweight people. Notably, China has the largest number of overweight people (more than 89.6 million in 2014) [21]. Problems related to obesity have attracted an increasing amount of attention from surgical specialists since a large amount of adipose tissue in the abdominal wall and the abdominal cavity greatly increases the difficulty of exposing the operative field in laparotomy [4]. Studies also indicate that, compared with non-obese patients, obese patients experience significantly longer operation times, more intraoperative blood loss, and are more likely to develop postoperative complications after abdominal operation $[4,20]$.

Table 3. Univariate and multivariate logistic analysis of factors associated with total postoperative complications

\begin{tabular}{|c|c|c|c|c|c|c|c|c|}
\hline \multirow[t]{3}{*}{ Factors } & \multicolumn{4}{|c|}{ Unmatched comparison } & \multicolumn{4}{|l|}{ Matched comparison } \\
\hline & \multicolumn{2}{|c|}{ Univariate analysis } & \multicolumn{2}{|l|}{ Multivariate analysis } & \multicolumn{2}{|l|}{ Univariate analysis } & \multicolumn{2}{|l|}{ Multivariate analysis } \\
\hline & OR $(95 \% \mathrm{CI})$ & $P$ & OR $(95 \% \mathrm{CI})$ & $P$ & OR $(95 \% \mathrm{CI})$ & $P$ & OR $(95 \%$ CI $)$ & $P$ \\
\hline \multicolumn{9}{|l|}{ Gender } \\
\hline Male & Ref & & & & Ref & & & \\
\hline Female & $0.984(0.525-1.846)$ & 0.118 & & & $2.291(0.809-6.484)$ & 0.118 & & \\
\hline \multicolumn{9}{|l|}{ Age (y) } \\
\hline$\leq 65$ & Ref & & & & Ref & & & \\
\hline$>65$ & $1.847(1.065-3.204)$ & 0.029 & & & $1.514(0.632-3.628)$ & 0.352 & & \\
\hline \multicolumn{9}{|l|}{ BMI $\left(\mathrm{kg} / \mathrm{m}^{2}\right)$} \\
\hline $18.5-24$ & Ref & 0.365 & & & Ref & 0.212 & & \\
\hline$>24$ & $0.780(0.456-1.335)$ & & & & $0.571(0.237-1.376)$ & & & \\
\hline \multicolumn{9}{|l|}{ NRS 2002 score } \\
\hline $1-2$ & Ref & & & & Ref & & & \\
\hline $3-4$ & $1.234(0.667-2.283)$ & 0.503 & & & $1.297(0.437-3.849)$ & 0.640 & & \\
\hline $5-6$ & $2.587(0.944-7.094)$ & 0.065 & & & $1.405(0.121-16.305)$ & 0.786 & & \\
\hline \multicolumn{9}{|l|}{ ASA grade } \\
\hline $1-2$ & Ref & & & & Ref & & & \\
\hline $3-4$ & 1.179 (0.615-2.259) & 0.621 & & & $0.736(0.143-3.778)$ & 0.714 & & \\
\hline \multicolumn{9}{|l|}{ Hypertension } \\
\hline No & Ref & & & & Ref & & & \\
\hline Yes & 0.967 (0.555-1.683) & 0.905 & & & $0.898(0.369-2.181)$ & 0.811 & & \\
\hline \multicolumn{9}{|l|}{ Diabetes mellitus } \\
\hline No & Ref & & & & Ref & & & \\
\hline Yes & 1.369 (0.715-2.619) & 0.343 & & & $0.714(0.213-2.390)$ & 0.585 & & \\
\hline \multicolumn{9}{|c|}{ Previous abdominal surgery } \\
\hline No & Ref & & & & Ref & & & \\
\hline Yes & $1.014(0.4518-2.281)$ & 0.973 & & & $0.420(0.048-3.652)$ & 0.432 & & \\
\hline \multicolumn{9}{|l|}{ Tumor location } \\
\hline Cardia & Ref & & & & Ref & & & \\
\hline Body & $0.459(0.186-1.131)$ & 0.091 & & & $0.286(0.061-1.328)$ & 0.110 & & \\
\hline Antrum & $0.593(0.288-1.225)$ & 0.158 & & & $0.470(0.127-1.733)$ & 0.257 & & \\
\hline Total & $0.412(0.073-2.307)$ & 0.313 & & & & 1.000 & & \\
\hline \multicolumn{9}{|l|}{ Differentiated degree } \\
\hline Differentiated & Ref & & & & Ref & & & \\
\hline Undifferentiated & $0.844(0.332-2.143)$ & 0.721 & & & $0.300(0.036-2.521)$ & 0.208 & & \\
\hline Signet ring carcinoma & $1.211(0.571-2.566)$ & 0.617 & & & $1.718(0.585-5.047)$ & 0.325 & & \\
\hline \multicolumn{9}{|l|}{ Pathological type } \\
\hline Ulcerative type & Ref & & & & Ref & & & \\
\hline Non-ulcerative type & $1.414(0.600-3.331)$ & 0.428 & & & $0.736(0.143-3.778)$ & 0.714 & & \\
\hline \multicolumn{9}{|l|}{ TNM stage } \\
\hline I & Ref & & & & Ref & & & \\
\hline II & $1.487(0.741-2.984)$ & 0.264 & & & $1.929(0.667-5.578)$ & 0.226 & & \\
\hline III & $1.190(0.632-2.240)$ & 0.589 & & & $0.875(0.301-2.540)$ & 0.803 & & \\
\hline \multicolumn{9}{|c|}{ Combined organ resection } \\
\hline No & Ref & & & & Ref & & & \\
\hline Yes & $1.113(0.396-3.124)$ & 0.839 & & & $1.333(0.116-15.311)$ & 0.817 & & \\
\hline Laparoscopic gastrect & & & & & & & & \\
\hline No & Ref & & Ref & & Ref & & Ref & \\
\hline Yes & $0.216(0.093-0.501)$ & $<0.001$ & $0.215(0.087-0.530)$ & 0.001 & $0.176(0.064-0.484)$ & 0.001 & $0.141(0.042-0.472)$ & 0.001 \\
\hline
\end{tabular}

BMI body mass index; ASA American Society of Anesthesiologists, NRS 2002 nutritional risk screening 2002, TNM tumor-node-metastasis.

*Statistically significant $(\mathrm{P}<0.05)$. 
Table 4. Univariate and multivariate Cox regression analysis of factors associated with overall survival

\begin{tabular}{|c|c|c|c|c|c|c|c|c|}
\hline \multirow[t]{3}{*}{ Factors } & \multicolumn{4}{|l|}{ Unmatched comparison } & \multicolumn{4}{|l|}{ Matched comparison } \\
\hline & \multicolumn{2}{|l|}{ Univariate analysis } & \multicolumn{2}{|l|}{ Univariate analysis } & \multicolumn{2}{|l|}{ Univariate analysis } & \multicolumn{2}{|l|}{ Univariate analysis } \\
\hline & HR (95\% CI) & $P$ & HR $(95 \%$ CI) & $P$ & $\mathrm{HR}(95 \% \mathrm{CI})$ & $P$ & HR $(95 \% \mathrm{CI})$ & $P$ \\
\hline \multicolumn{9}{|l|}{ Gender } \\
\hline Male & Ref & & & & Ref & & & \\
\hline Female & $0.908(0.318-2.773)$ & 0.908 & & & $0.923(0.265-3.214)$ & 0.900 & & \\
\hline \multicolumn{9}{|l|}{ Age (y) } \\
\hline$\leq 65$ & Ref & & & & Ref & & & \\
\hline$>65$ & $1.083(0.483-2.420)$ & 0.846 & & & $2.320(0.857-6.276)$ & 0.098 & & \\
\hline \multicolumn{9}{|l|}{ BMI $\left(\mathrm{kg} / \mathrm{m}^{2}\right)$} \\
\hline $18.5-24$ & Ref & & & & Ref & & & \\
\hline$>24$ & $0.710(0.328-1.538)$ & 0.385 & & & $2.222(0.783-6.311)$ & 0.134 & & \\
\hline \multicolumn{9}{|l|}{ NRS 2002 score } \\
\hline $1-2$ & Ref & & Ref & & Ref & & Ref & \\
\hline $3-4$ & 1.687 (0.719-3.955) & 0.229 & $1.142(0.470-2.771)$ & 0.770 & $1.698(0.540-5.341)$ & 0.366 & $2.588(0.579-11.570)$ & 0.213 \\
\hline $5-6$ & $3.106(1.061-9.091)$ & $0.039^{*}$ & $1.647(0.533-5.083)$ & 0.386 & $6.180(1.301-28.055)$ & $0.018^{*}$ & $12.968(1.780-94.495)$ & $0.011^{*}$ \\
\hline \multicolumn{9}{|l|}{ ASA grade } \\
\hline $1-2$ & Ref & & & & Ref & & & \\
\hline $3-4$ & $1.217(0.497-2.983)$ & 0.667 & & & $2.085(0.599-7.257)$ & 0.248 & & \\
\hline \multicolumn{9}{|l|}{ Hypertension } \\
\hline No & Ref & & & & Ref & & & \\
\hline Yes & $0.507(0.229-1.123)$ & 0.094 & & & $0.743(0.275-2.010)$ & 0.559 & & \\
\hline \multicolumn{9}{|l|}{ Diabetes mellitus } \\
\hline No & Ref & & & & Ref & & & \\
\hline Yes & $0.418(0.143-1.226)$ & 0.112 & & & $0.959(0.275-3.342)$ & 0.948 & & \\
\hline \multicolumn{9}{|l|}{$\begin{array}{l}\text { Previous abdominal } \\
\text { surgery }\end{array}$} \\
\hline No & Ref & & & & Ref & & & \\
\hline Yes & $1.643(0.612-4.408)$ & 0.324 & & & $0.829(0.110-6.256)$ & 0.856 & & \\
\hline \multicolumn{9}{|l|}{ Tumor location } \\
\hline Cardia & Ref & & & & Ref & & & \\
\hline Body & $1.102(0.274-4.435)$ & 0.891 & & & $1.071(0.208-5.521)$ & 0.935 & & \\
\hline Antrum & $1.182(0.391-3.569)$ & 0.767 & & & $0.717(0.155-3.323)$ & 0.671 & & \\
\hline Total & $3.909(0.699-21.861)$ & 0.121 & & & $6.520(0.582-72.979)$ & 0.128 & & \\
\hline \multicolumn{9}{|l|}{ Differentiated degree } \\
\hline Differentiated & Ref & & & & Ref & & & \\
\hline Undifferentiated & $1.214(0.400-3.680)$ & 0.732 & & & $1.310(0.293-5.864)$ & 0.724 & & \\
\hline Signet ring carcinoma & $1.257(0.460-3.433)$ & 0.655 & & & $1.033(0.291-3.601)$ & 0.960 & & \\
\hline \multicolumn{9}{|l|}{ Pathological type } \\
\hline Ulcerative type & Ref & & & & Ref & & & \\
\hline Non-ulcerative type & $0.865(0.256-2.925)$ & 0.816 & & & $1.732(0.395-7.596)$ & 0.467 & & \\
\hline \multicolumn{9}{|l|}{ TNM stage } \\
\hline I & Ref & & Ref & & Ref & & Ref & \\
\hline II & $9.633(1.154-80.384)$ & $0.036^{*}$ & 9.815 (1.174-82.049) & $0.035^{*}$ & $4.758(0.871-25.995)$ & 0.072 & $5.686(0.737-43.861)$ & 0.095 \\
\hline III & $14.208(1.898-106.340)$ & $0.010^{*}$ & 10.735 (1.386-83.148) & $0.023^{*}$ & $10.492(2.321-47.434)$ & $0.002^{*}$ & $17.492(2.445-125.157)$ & $0.004^{*}$ \\
\hline \multicolumn{9}{|c|}{ Combined organ resection } \\
\hline No & Ref & & & & Ref & & & \\
\hline Yes & $1.630(0.381-6.969)$ & 0.510 & & & $2.425(0.321-18.315)$ & 0.391 & & \\
\hline Laparoscopic gastrecto & & & & & & & & \\
\hline No & Ref & & Ref & & Ref & & Ref & \\
\hline Yes & $0.135(0.018-1.002)$ & $0.050^{*}$ & $0.178(0.024-1.343)$ & 0.094 & $0.280(0.091-0.859)$ & $0.026^{*}$ & $0.264(0.077-0.905)$ & $0.034^{*}$ \\
\hline
\end{tabular}

Considering the priority of $\mathrm{VO}$ over BMI in several studies [11, 20], we used CT-based-VFA for determining $\mathrm{VO}$ in this study and observed a high incidence of $\mathrm{VO}$ in patients with GC (42.61\%). Therefore, it is necessary to focus on the clinical effect of $\mathrm{VO}$ in patients with GC. Additionally, although several studies $[22,23]$ have already shown that LG is superior to OG in short-term outcomes, no such studies focus on whether the minimal invasiveness of laparoscopic GC surgery can reduce the surgical stress on obese patients, improve their tolerance to surgery, or improve their short- and long-term outcomes.

In the present study, patients in the LG group experienced fewer postoperative complications and shorter postoperative hospital stays than patients in the OG group. Additionally, LG turned out to be the only independent protective factor for total postoperative complications, which was similar to the results of a recent study which demonstrated that the 
overall incidence of complications from laparoscopic-assisted surgery was significantly lower than that from open surgery in colorectal cancer patients with VO [24]. Additionally, a previous observational study also showed that hand-assisted laparoscopic distal gastrectomy had obvious superiority over open distal gastrectomy in reducing estimated blood loss, wound length, number of analgesic injections, time to the first flatus, and postoperative hospital stay in obese patients [25]. Another interesting finding was that all the examined complications were lower in the laparoscopic group than in the open group; however, only the difference in the incidence of surgical complications was statistically significant, which may be owing to the small sample size. Previous studies [26, 27] have indicated that the duration of the operation and the volume of blood loss are greatly associated with morbidity after gastrectomy. Owing to the lack of tactile sensation, a narrow operating field, a complicated vascular structure in the splenic hilum, and the advanced techniques of systemic lymph node dissections, LG is a time-consuming procedure. However, considering that VO significantly increases insulin resistance, decreases oxygen tension within surgical wounds, impairs tissue penetration of perioperative antibiotics, and increases operative blood loss, LG, which has the advantage of less trauma, smaller wounds, and reduced blood loss [28], still has obvious superiority over OG in patients with VO.

Another major concern in performing LG is the long-term survival of patients with GC. As the oncological outcomes of LG are comparable to those associated with OG, studies have revealed that LG could yield similar oncologic outcomes to $O G$ in treating GC $[14,29,30]$. However, the relationship between LG and OG in terms of the long-term efficacy in patients with $\mathrm{VO}$ has not yet been reported. Interestingly, our results first demonstrated that OS was significantly longer in patients with $\mathrm{VO}$ who underwent LG, and laparoscopic surgery was an independent protective factor for OS. It is worth mentioning that LG was not significantly related to OS in unmatched comparison. That was probably because the effect of LG was masked by the difference of these long-term survival-related factors such as tumor staging, age, and nutrition score. However, in the matched comparison, where there were no other differences between the two groups, LG turned out to be significantly related to the OS. This was probably because compared with open surgery, LG has a better surgical vision and a larger cleaning scope for these visceral obesity patients. On the other hand, although these patients were well matched in this study, patients seemed to be younger and lower TNM staging, which also contributed to the active role of LG for favorable long-term outcomes in GC patients with VO. In addition, our findings suggest that the TNM stage and NRS score were also independent prognostic factors for OS, which is similar to the traditional knowledge on the prognostic factors for survival of patients with GC.

To the best of our knowledge, the present study is the first to evaluate the short- and long-term outcomes of LG in GC patients with VO using a PSM analysis. However, we recognize that our study has several limitations. First and most important, this is not a randomized controlled trial, and inherent selection biases can be adjusted but cannot entirely be eliminated by using PSM. Second, this was not a multiple-center study; therefore, our results may not be directly applicable to other populations. Third, a part of the patients went to other medical centers for further treatment, and a few others did not undergo regular out-patient review. Therefore, we could only obtain the time of patient's death by phone and have no information about the recurrence time. As a result, the analysis of disease-free survival (DFS) and progression-free survival (PFS) was only partially complete. Finally, the small sample size after matching may limit the broader applicability of our findings, and a large-sample study is still necessary.

In conclusion, compared with OG, LG can significantly reduce the incidence of postoperative complications in patients with $\mathrm{VO}$, promote postoperative recovery, and provide better long-term efficacy. Therefore, laparoscopic surgery can be strongly recommended surgical for GC patients with VO.

\section{Acknowledgements}

This study was funded by the National Natural Science Foundation of China (grant no. 31670922 and 81672707), the Zhejiang general scientific research project of the education department (grant no. Y201941489), and the Wenzhou Basic Scientific Research Projects (grant no. Y20180064). We thank Editage Group (https://www.editage.cn/) for polishing the draft of this manuscript.

\section{Ethical disclosure}

The study was approved by the ethics committees of the Second Affiliated Hospital of Wenzhou Medical University, and all participants provided written informed consent prior to study participation. 


\section{Competing Interests}

The authors have declared that no competing interest exists.

\section{References}

1. Torre LA, Bray F, Siegel RL, Ferlay J, Lortet-Tieulent J, et al. Global cancer statistics, 2012. CA Cancer J Clin 2015;65:87-108.

2. Ferlay J, Soerjomataram I, Dikshit R, Eser S, Mathers C, et al. Cancer incidence and mortality worldwide: sources, methods and major patterns in GLOBOCAN 2012. Int J Cancer 2015;136:E359-86.

3. Go JE, Kim MC, Kim KH, Oh JY, Kim YM. Effect of visceral fat area on outcomes of laparoscopyassisted distal gastrectomy for gastric cancer: subgroup analysis by gender and parameters of obesity. Ann Surg Treat Res 2015;88:318-324

4. Liu M, Xing J, Arslan A, Tan F, Fan Y, et al. Safety and efficacy of laparoscopic gastrectomy in obese patients with gastric cancer. Medicine (Baltimore) 2019;98:e17991.

5. Lee WJ, Wang W, Chen TC, Wei PL, Lin CM, et al. Clinical significance of central obesity in laparoscopic bariatric surgery. Obes Surg 2003:13:921-5.

6. Hagiwara M, Miyajima A, Hasegawa M, Jinzaki M, Kikuchi E, et al. Visceral obesity is a strong predictor of perioperative outcome in patients undergoing laparoscopic radical nephrectomy. BJU Int 2012;110:E980-4.

7. Pou KM, Massaro JM, Hoffmann U, Vasan RS, Maurovich-Horvat P, et al. Visceral and subcutaneous adipose tissue volumes are cross-sectionally related to markers of inflammation and oxidative stress: the Framingham Heart Study. Circulation 2007;116:1234-1241.

8. Shiomi A, Kinugasa Y, Yamaguchi T, Kagawa H, Yamakawa Y. Robot-assisted versus laparoscopic surgery for lower rectal cancer: the impact of visceral obesity on surgical outcomes. Int J Colorectal Dis 2016;31:1701-1710.

9. Kang J, Baek SE, Kim T, Hur H, Min BS, et al. Impact of fat obesity on laparoscopic total mesorectal excision: more reliable indicator than body mass index. Int J Colorectal Dis 2012;27:497-505.

10. Cakir $\mathrm{H}$, Heus $\mathrm{C}$, Verduin WM, Lak A, Doodeman HJ, et al. Visceral obesity, body mass index and risk of complications after colon cancer resection: A retrospective cohort study. Surgery 2015;157:909-915.

11. Yang SJ, Li HR, Zhang WH, Liu K, Zhang DY, et al. Visceral Fat Area (VFA) Superior to BMI for Predicting Postoperative Complications After Radical Gastrectomy: a Prospective Cohort Study. J Gastrointest Surg 2020; 24:12981306.

12. Kitano S, Iso Y, Moriyama M, Sugimachi K. Laparoscopy-assisted Billroth I gastrectomy. Surg Laparosc Endosc 1994;4:146-8.

13. Zheng HL, Lu J, Zheng CH, Li P, Xie JW, et al. Short- and Long-Term Outcomes in Malnourished Patients After Laparoscopic or Open Radical Gastrectomy. World J Surg 2018;42:195-203.

14. Li HZ, Chen JX, Zheng Y, Zhu XN. Laparoscopic-assisted versus open radical gastrectomy for resectable gastric cancer: Systematic review, meta-analysis, and trial sequential analysis of randomized controlled trials. J Surg Oncol 2016;113:756-67.

15. Zeng F, Chen L, Liao M, Chen B, Long J, et al. Laparoscopic versus open gastrectomy for gastric cancer. World J Surg Oncol 2020;18:20.

16. Dindo D, Demartines N, Clavien PA. Classification of surgical complications: a new proposal with evaluation in a cohort of 6336 patients and results of a survey. Ann Surg 2004:240:205-13.

17. Chen XD, Mao CC, Zhang WT, Lin J, Wu RS, et al. A quantified risk-scoring system and rating model for postsurgical gastroparesis syndrome in gastric cancer patients. J Surg Oncol 2017;116:533-544.

18. Shuster A, Patlas M, Pinthus JH, Mourtzakis M. The clinical importance of visceral adiposity: a critical review of methods for visceral adipose tissue analysis. Br J Radiol 2012;85:1-10.

19. Borkan GA, Gerzof SG, Robbins AH, Hults DE, Silbert CK, et al. Assessment of abdominal fat content by computed tomography. Am J Clin Nutr 1982;36:172-7.

20. Watanabe J, Tatsumi K, Ota M, Suwa Y, Suzuki S, et al. The impact of visceral obesity on surgical outcomes of laparoscopic surgery for colon cancer. Int J Colorectal Dis 2014;29:343-51.

21. Collaboration NCDRF. Trends in adult body-mass index in 200 countries from 1975 to 2014: a pooled analysis of 1698 population-based measurement studies with 19.2 million participants. Lancet 2016;387:1377-1396.

22. Takiguchi S, Fujiwara Y, Yamasaki M, Miyata H, Nakajima K, et al. Laparoscopy-assisted distal gastrectomy versus open distal gastrectomy. A prospective randomized single-blind study. World J Surg 2013;37:2379-86.

23. Kim W, Kim HH, Han SU, Kim MC, Hyung WJ, et al. Decreased Morbidity of Laparoscopic Distal Gastrectomy Compared With Open Distal Gastrectomy for Stage I Gastric Cancer: Short-term Outcomes From a Multicenter Randomized Controlled Trial (KLASS-01). Ann Surg 2016;263:28-35.

24. Ye XZ, Chen XY, Ruan XJ, Chen WZ, Ma LL, et al. Laparoscopic-assisted colorectal surgery benefits visceral obesity patients: a propensity-matched analysis. Eur J Gastroenterol Hepatol 2019;31:786-791.

25. Zhang GT, Liang D, Zhang XD. Comparison of hand-assisted laparoscopic and open radical distal gastrectomy for obese patients. Am Surg 2013;79:1273-8.
26. Sugimoto M, Kinoshita T, Shibasaki H, Kato Y, Gotohda N, et al. Short-term outcome of total laparoscopic distal gastrectomy for overweight and obese patients with gastric cancer. Surg Endosc 2013;27:4291-6.

27. Noshiro H, Shimizu S, Nagai E, Ohuchida K, Tanaka M. Laparoscopy-assisted distal gastrectomy for early gastric cancer: is it beneficial for patients of heavier weight? Ann Surg 2003;238:680-5.

28. Yang T, Wei M, He Y, Deng X, Wang Z. Impact of visceral obesity on outcomes of laparoscopic colorectal surgery: a meta-analysis. ANZ J Surg 2015;85:507-513.

29. Wang H, Mou T, Chen H, Hu Y, Lin T, et al. Long-term outcomes of laparoscopy-assisted distal gastrectomy versus open distal gastrectomy for gastric cancer: a 10-year single-institution experience. Surg Endosc 2019;33:135-144.

30. Aratani K, Sakuramoto S, Chuman M, Kasuya M, Wakata M, et al. Laparoscopy-assisted Distal Gastrectomy for Gastric Cancer in Elderly Patients: Surgical Outcomes and Prognosis. Anticancer Res 2018;38:1721-1725. 